a cap on the state of) the uppermost region of the unhydrated mantle, unless it represents a local phenomenon.

The global surface-water inventory of Mars was originally estimated to be about $2 \times 10^{7}$ to $2 \times 10^{8} \mathrm{~km}^{3}$ (ref. 7 , and references therein), on the basis of the size of the putative ancient oceans. But estimates of the total water loss to space are much smaller (less than $10^{7} \mathrm{~km}^{3}$, based on atmospheric-escape models ${ }^{7}$ ). The discrepancy between these estimates hints at the existence of a 'missing' water reservoir beneath the surface. The widespread distribution of hydrated materials on the surface of Mars also implies the existence of a crustal water reservoir, but conventional spectroscopic observations are able to see only the surface veneer. Wade and colleagues' thermodynamic modelling, together with remotesensing observations, offers a means to work out the depth profile of hydrated materials, and to calculate a reasonable estimate of the volume of the crustal water reservoir.

Ground ice might also account for the missing water reservoir on Mars ${ }^{8-10}$. Subsurface radar-sounder measurements ${ }^{8}$ have detected an anomaly in an electrical property of rocks in the planet's northern hemisphere, which implies that massive ice deposits are embedded among or between layers of sediment and volcanic materials at a depth of $60-80 \mathrm{~m}$. The ground-ice model has also been proposed on the basis of analyses of hydrogen isotopes in Martian meteorites ${ }^{9}$ and of crater morphology ${ }^{10}$. The crater study indicates that the subsurface water ice has a volume of about $3 \times 10^{7} \mathrm{~km}^{3}$, which is comparable to the size of the ancient oceans. Subsurface exploration will be required to test both the hydratedcrust and ground-ice theories, and therefore to shed light on the evolution of the Martian water inventory.

Tomohiro Usui is at the Earth-Life Science
Institute, Tokyo Institute of Technology,

Tokyo 152-8551, Japan.

e-mail:tomohirousui@elsi.jp

1. Lammer, H. et al. Space Sci. Rev. 174, 113-154 (2013).

2. Wade, J., Dyck, B., Palin, R. M., Moore, J. D. P. \& Smye, A. J. Nature 552, 391-394 (2017).

3. Herd, C. D. K. et al. Geochim. Cosmochim. Acta 66, 2025-2036 (2002).

4. Usui, T., Alexander, C. M. O'D., Wang, J., Simon, J. I. \& Jones, J. H. Earth Planet. Sci. Lett. 357-358, 119-129 (2012).

5. Saal, A. E., Hauri, E. H., Langmuir, C. H. \& Perfit, M. R. Nature 419, 451-455 (2002).

6. Filiberto, J. \& Dasgupta, R. J. Geophys. Res. Planets 120, 109-122 (2015).

7. Kurokawa, H. et al. Earth Planet. Sci. Lett. 394, 179-185 (2014).

8. Mouginot, J., Pommerol, A., Beck, P., Kofman, W. \& Clifford, S. M. Geophys. Res. Lett. 39, L02202 (2012).

9. Usui, T., Alexander, C. M. O’D., Wang, J., Simon, J. I. \& Jones, J. H. Earth Planet. Sci. Lett. 410, 140-151 (2015).

10.Weiss, D. K. \& Head, J. W. Icarus 288, 120-147 (2017).

\title{
Tumour lymph vessels boost immunotherapy
}

A high level of expression of the growth-factor protein VEGF-C is associated with tumours that have extensive lymph vessels and poor prognosis. It emerges that such tumours are highly susceptible to immunotherapy.

\section{CHRISTINE MOUSSION \\ \& SHANNON J. TURLEY}

A dvances in our understanding of tumour immunology have led to immense clinical interest in harnessing immune cells to target cancer ${ }^{1}$. The immune system's killer (cytotoxic) T cells can search for and destroy abnormal cells that they encounter while patrolling tissues. However, in the suppressive microenvironment of a tumour, such cells often enter a dysfunctional state. A goal of cancer immunotherapy is to trigger or revitalize the antitumour immune response either through vaccination strategies or by using an approach called checkpoint-blockade therapy that dampens immuno-inhibitory signals, such as those of the PD-1 or CTLA-4 pathways ${ }^{2}$. Writing in Science Translational Medicine, Fankhauser et al. ${ }^{3}$ show that an increase in the expression of a protein that promotes tumour growth can also make a tumour more responsive to immunotherapy.

Tumours need a vascular supply to grow. Blood vessels bring nutrients and oxygen to a tumour, whereas lymphatic vessels remove fluid and waste. A tumour and the cells in its surrounding milieu stimulate the development

of these two branches of the vascular system by secreting proteins that are members of the vascular endothelial growth factor (VEGF) family ${ }^{4}$.

The protein VEGF-A promotes the generation of new blood vessels (angiogenesis). Decades of work aimed at impairing tumour growth by blocking angiogenesis has led to the approval of drugs that inhibit signalling by members of the VEGF family, with most approaches $^{4}$ target-

"Anincrease in the expression of a protein that promotes tumour growth can also make a tumour more responsive to immunotherapy.' ing VEGF-A.

VEGF-C promotes lymphatic-vessel formation (lymphangiogenesis) by signalling through the VEGFR3 receptor ${ }^{5}$. Lymphatic vessels can promote the process of metastasis - tumourcell spread beyond the primary tumour growth site - by providing a route for cancer cells to exit a tumour and reach nearby structures termed lymph nodes. This process enables tumour cells to grow in lymph nodes, and to disseminate from there to other locations. Many tumours associated with metastasis, such as melanoma and breast cancer, express high levels of VEGF-C and contain a dense network of lymphatic vessels ${ }^{6}$. Moreover, an increase in VEGF-C expression in tumours is highly correlated with metastasis to lymph nodes, and with a reduction in survival in individuals with different tumour types, including skin, breast and lung cancers ${ }^{7}$.

Does an increase in tumour lymphangiogenesis always promote tumour growth? Fankhauser and colleagues addressed this question by analysing the role of VEGF-Cdriven lymphangiogenesis in tumour growth. They studied mice that express high levels of VEGF-C and provide a model system for studying cancer. When the researchers treated these animals with VEGF-C-blocking antibodies, they observed a surprising result: although high VEGF-C expression is associated with poor tumour prognosis, VEGF-C inhibition in the context of tumour immunotherapy resulted in increased tumour growth. This suggested that VEGF-C might have another, previously unsuspected role in limiting tumour growth by boosting patient responses to immunotherapy.

The authors investigated the basis of this phenomenon. They found a strong positive correlation between VEGF-C levels and the strength of a tumour-specific cytotoxic T-cell response after immunotherapy in their model mice. They also observed a similar association between high VEGF-C expression and boosted immunotherapy responses (Fig. 1) when analysing data from people who have a metastatic form of skin cancer called melanoma. Together, these results suggest that VEGF-C and the lymphatic system might have unexpected roles in aiding cancer immunotherapy. Perhaps VEGF-C levels could be used as a biomarker to identify patients who are likely to respond to immunotherapy. 


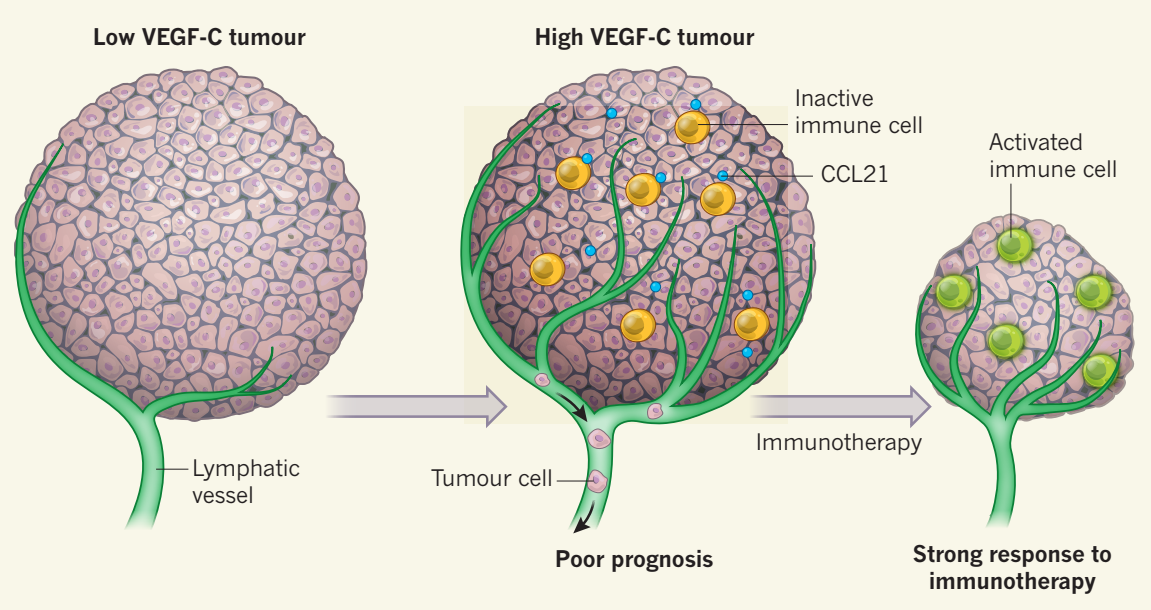

Figure 1 | Lymph-vessel formation and tumour response to immunotherapy. Fankhauser et al. ${ }^{3}$ studied the role of lymphatic vessels - the vasculature that provides a drainage system to remove waste from tissues - in the response of tumours to immunotherapy by using mouse models and analysing clinical data. Tumours that express low levels of the growth-factor protein VEGF-C do not have an extensive network of lymph vessels. However, if VEGF-C expression becomes high in a tumour, a dense network of lymph vessels forms. High VEGF-C levels are associated with poor prognosis. One possible reason for this is that lymph vessels provide a route for tumour-cell migration to other locations in the body. VEGF-C drives the expression of the protein CCL21, and Fankhauser and colleagues observed that the presence of CCL21 was associated with the infiltration of inactive immune cells into the tumour. If these tumours were then treated with immunotherapy to boost the immune response, the immune cells were activated and there was a strong tumour response to immunotherapy.

To understand the biology underlying this phenomenon, the authors investigated how the VEGF-C pathway affects immunotherapy responses. Using genetically engineered mice, they demonstrated that VEGF-C signalling, along with expansion of the lymphatic network at the tumour site, enhances the response to many types of immunotherapy, including cancer vaccines and checkpoint blockade. The authors observed that lymphangiogenic tumours with high VEGF-C expression fostered potent antitumour immune responses, inhibiting primary or metastatic tumour growth.

High VEGF-C expression in the authors' mouse model drove strong production of the protein CCL21. This protein is not usually expressed by tumours. It is expressed by cells that form the blood vessels and the surrounding material known as the stroma ${ }^{8}$. These are both associated with the body's lymphoidorgan sites, in which immune cells are initially activated during an immune response.

CCL21 acts as a type of chemoattractant called a chemokine ${ }^{9}$, that binds the CCR7 receptor on naive $\mathrm{T}$ cells (immune cells that have not yet been activated to mount an immune response) and drives the cells to migrate towards greater concentrations of the chemokine. The migration of naive T cells towards higher concentrations of CCL21 enables the cells to exit the bloodstream and enter lymphoid organs that are rich in CCL21. This is a necessary prelude to their activation and participation in an immune response.

The authors observed that their mice with high VEGF-C expression had a high level of naive $\mathrm{T}$ cells present within the tumours, and the number of naive $\mathrm{T}$ cells could be reduced if the animals were treated with antibodies that block CCR7 activity. Their analysis suggests that CCL21-enriched tumours might mimic lymphoid organs in a way that attracts naive T cells.

Although not addressed by Fankhauser and colleagues, it is interesting to consider whether VEGF-C signalling in tumours might promote the formation of tertiary lymphoid structures $^{10}$. These are formed from collections of immune cells clustered in structures that have lymph-node-like characteristics. Unusually high levels of CCL21 expression in tissues can be accompanied by formation of tertiary lymphoid structures that have an organized stromal component and specialized blood vessels named high endothelial venules ${ }^{11}$. These enable the recruitment of naive $\mathrm{T}$ cells from the bloodstream into the tissue and are thought to enhance local immune responses ${ }^{11}$. Because such structures can develop locally within tissues and bypass the need for the lymph node to activate immune cells, their formation in or near tumours might bolster anti-tumour immune responses and improve responsiveness to immunotherapy.

Therapeutics targeting the VEGF family in combination with immunotherapies might increase the tumour response to treatment

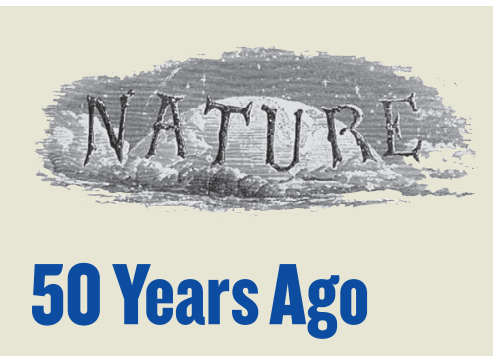

The decision of the council of the Zoological Society to ban all feeding by visitors at London Zoo and Whipsnade Park from the beginning of 1968 is the final stage of the policy adopted in 1965 in the interests of the health and general welfare of the animals ... The visitors too will be safer when the ban is enforced; during one year at London 180 people have been bitten by monkeys, parrots, cockatoos, ponies and other animals. During the same year elephants at London seized fourteen coats, twelve handbags, ten cameras, eight gloves and six return tickets to Leicester, damaging them all beyond repair ... The problem of educating the public to resist throwing buns, nuts and other food to the animals is considerable... Although nobody will be turned out of the zoo for feeding the animals, offenders will be asked to stop by watchful keepers. From Nature 23 December 1967

\section{Years Ago}

A very great advance was made in the earliest form of electricity generator by the late James Wimshurst in the year 1882. At that time several forms of the Holtz and Voss machines were in use, but their behaviour was most erratic ... Wimshurst succeeded in producing a machine that would "excite" with certainty under almost any atmospheric condition, and by combining a number of plates was able greatly to increase the output... The uses of the machine are very numerous. It has been employed with success in agricultural experiments, where greatly increased plant growth under the influence of the static discharge has been recorded. In electro-therapy its value is fully recognised, and the static discharge is now in constant use in many hospitals.

From Nature 27 December 1917 
by increasing the formation of tertiary lymphoid structures and the recruitment of naive $T$ cells, as indicated in mouse models in which combinations of VEGF-A inhibition and checkpoint blockade were tested ${ }^{12}$. Fankhauser and colleagues raise the provocative idea that lymphatic vessels and active VEGFR3 signalling driven by VEGF-C might be beneficial for individuals with cancer who are being treated with immunotherapy. If this is the case, successful strategies may need to combine vascular-modulating drugs with cancer immunotherapeutics that preserve lymphangiogenic features.

Christine Moussion and Shannon J. Turley are at Genentech, South San Francisco, California 94080, USA.

e-mail:turley.shannon@gene.com
1. Chen, D. S. \& Mellman, I. Nature 541, 321-330 (2017).

2. Topalian, S. L., Drake, C. G. \& Pardoll, D. M. Cancer Cell 27, 450-461 (2015).

3. Fankhauser, M. et al. Sci. Transl. Med. 9, eaal4712 (2017).

4. Chung, A. S., Lee, J. \& Ferrara, N. Nature Rev. Cancer 10, 505-514 (2010).

5. Adams, R. H. \& Alitalo, K. Nature Rev. Mol. Cell Biol. 8, 464-478 (2007).

6. Skobe, M. et al. Nature Med. 7, 192-198 (2001)

7. Stacker, S. et al. Nature Rev. Cancer 14, 159-172 (2014).

8. Förster, R., Davalos-Misslitz, A. C. \& Rot, A. Nature Rev. Immunol. 8, 362-371 (2008).

9. Issa, A., Le, T. X., Shoushtari, A. N., Shields, J. D. \& Swartz, M. A. Cancer Res. 69, 349-357 (2009). 10. Luther. S. A. et al. J. Immunol. 169, 424-433 (2002).

11.Dieu-Nosjean, M.-C., Goc, J., Giraldo, N. A. Sautès-Fridman, C. \& Fridman, W. H. Trends Immunol. 35, 571-580 (2014).

12.Allen, E. et al. Sci. Transl. Med. 9, eaak9679 (2017).

\section{NEURODEGENERATIVE DISORDERS}

\section{Specks of insight into Alzheimer's disease}

Inflammatory cues trigger microglial cells to release the protein ASC. It emerges that specks of ASC promote a hallmark of Alzheimer's disease in the brains of mice - aggregation and deposition of amyloid- $\beta$ protein. SEE ARTICLE P.355

\section{RICHARD M. RANSOHOFF}

$\mathrm{F}$ rom one perspective, Alzheimer's disease can be considered to be relatively simple: it requires the formation of aggregated clumps of the protein amyloid- $\beta$ in the brain, which promote a string of downstream consequences that lead to dementia. But when all contributing factors are taken into account, it is fearsomely complex. For instance, consider the potential role of inflammatory processes. There are many indicators of inflammation in Alzheimer's disease ${ }^{1}$ : levels of immune molecules are elevated; neuronsupporting cells called glia react strongly to the abnormal tissue environment; and changes arise in the specialized vasculature of the blood-brain barrier. Furthermore, subtle genetic alterations in glia can augment the risk of developing Alzheimer's disease ${ }^{2}$. But despite this evidence, details of how inflammation might promote the disease have been evasive ${ }^{1}$. On page 355 , Venegas et al. ${ }^{3}$ provide evidence that, in mice, an extracellular form of an immune-activation protein promotes amyloid- $\beta$ aggregation.

Amyloid- $\beta$ is derived from a larger protein, amyloid precursor protein (APP), which is cleaved to produce a family of shorter peptides. Alzheimer's disease can be caused directly by mutations in either APP or the enzymes that process it, if they lead to the production of versions of amyloid- $\beta$ that are prone to aggregating into soluble, cell-damaging clusters. These soluble amyloid- $\beta$ aggregates subsequently undergo further aggregation and are deposited in brain tissue in clumps called amyloid plaques. These groundbreaking findings undergird all research into the disease. But the specific mechanisms by which plaques form have been elusive. And given that, so far, all phase III clinical trials of prospective disease-modifying therapies have failed ${ }^{5,6}$, the identification of specific molecular players in plaque formation would be extremely welcome, because it could accelerate the development of better therapies.

Venegas et al. began from their group's previous observation ${ }^{7}$ that mice lacking the protein NLRP3 have some protection against amyloid- $\beta$ deposition. This finding was obtained by deleting the Nlrp3 gene in a mouse strain dubbed APP/PS1, which has been genetically engineered to express human genes that contain mutations associated with Alzheimer's disease; animals therefore show abundant deposition of aggregated amyloid- $\beta$ and cognitive impairment. NLRP3 is an immune sensor that, in the brain, is found in immunerelated cells of the central nervous system called microglia. It detects abnormal biochemical substances, including the products 